\title{
Maintenance erlotinib in advanced nonsmall cell lung cancer: cost-effectiveness in EGFR wild-type across Europe
}

This article was published in the following Dove Press journal:

ClinicoEconomics and Outcomes Research

13 September 2012

Number of times this article has been viewed

\author{
Silke Walleser ${ }^{\prime}$ \\ Joshua Ray ${ }^{2}$ \\ Helge Bischoff ${ }^{3}$ \\ Alain Vergnenègre ${ }^{4}$ \\ Hubertus Rosery ${ }^{5}$ \\ Christos Chouaid ${ }^{6}$ \\ David Heigener ${ }^{7}$ \\ Javier de Castro Carpeño ${ }^{8}$ \\ Marcello Tiseo' \\ Stefan Walzer ${ }^{2}$ \\ 'Health Economic Consultancy, \\ Renens, Switzerland; ${ }^{2} \mathrm{~F}$ Hoffmann-La \\ Roche Pharmaceuticals AG, Basel, \\ Switzerland; ${ }^{3}$ Thoracic Hospital of \\ Heidelberg, Heidelberg, Germany; \\ ${ }^{4}$ Limoges University Hospital, Limoges, \\ France; ${ }^{5}$ Assessment-in-Medicine \\ $\mathrm{GmbH}$, Loerrach, Germany; ${ }^{6} \mathrm{Hospital}$ \\ Saint Antoine, Paris, France; ${ }^{7}$ Hospital \\ Grosshansdorf, Grosshansdorf, \\ Germany; ${ }^{8}$ University Hospital La Paz, \\ Madrid, Spain; ' University Hospital \\ of Parma, Parma, Italy
}

Correspondence: Hubertus Rosery Assessment-in-Medicine $\mathrm{GmbH}$, Marie-Curie-Strasse 8, 79539 Loerrach, Germany

Tel +49 762। 705। 0510

$\mathrm{Fax}+49762170510530$

Email hubertus.rosery@assessment-inmedicine.de
Background: First-line maintenance erlotinib in patients with locally advanced or metastatic nonsmall cell lung cancer (NSCLC) has demonstrated significant overall survival and progression-free survival benefits compared with best supportive care plus placebo, irrespective of epidermal growth factor receptor (EGFR) status (SATURN trial). The cost-effectiveness of first-line maintenance erlotinib in the overall SATURN population has been assessed and published recently, but analyses according to EGFR mutation status have not been performed yet, which was the rationale for assessing the cost-effectiveness of first-line maintenance erlotinib specifically in EGFR wild-type metastatic NSCLC.

Methods: The incremental cost per life-year gained of first-line maintenance erlotinib compared with best supportive care in patients with EGFR wild-type stable metastatic NSCLC was assessed for five European countries (the United Kingdom, Germany, France, Spain, and Italy) with an area-under-the-curve model consisting of three health states (progression-free survival, progressive disease, death). Log-logistic survival functions were fitted to Phase III patient-level data (SATURN) to model progression-free survival and overall survival. The first-line maintenance erlotinib therapy cost (modeled for time to treatment cessation), medication cost in later lines, and cost for the treatment of adverse events were included. Deterministic and probabilistic sensitivity analyses using Monte Carlo simulation (1000 iterations) were performed.

Results: According to the model simulations, first-line maintenance erlotinib compared with best supportive care in EGFR wild-type stable metastatic NSCLC resulted in 4.57 months of life gained (17.82 months for erlotinib versus 13.24 months for best supportive care) and 1.14 months of life without progression gained (erlotinib 4.29 versus best supportive care 3.15), and incremental total costs of erlotinib from $€ 7897$ (UK) to $€ 9580$ (Germany). The corresponding mean incremental cost per life-year gained of erlotinib ranged between $€ 20,711$ (UK) and $€ 25,124$ (Germany). Sensitivity analyses confirmed these results.

Conclusion: First-line erlotinib maintenance treatment is cost-effective compared with best supportive care in EGFR wild-type stable metastatic NSCLC, irrespective of the country setting.

Keywords: nonsmall cell lung cancer, erlotinib, cost-benefit analysis, epidermal growth factor receptor, wild-type, Europe

\section{Introduction}

Lung cancer is the most frequently diagnosed malignancy in the world, and the leading cause of cancer-related death. ${ }^{1}$ Based on histology, lung cancer can be broadly divided into small cell lung cancer and nonsmall cell lung cancer (NSCLC). ${ }^{2,3}$ The latter accounts for about $80 \%$ of all lung cancer cases, ${ }^{1}$ and is divided into three major histological subtypes, ie, squamous cell carcinoma, adenocarcinoma, and large cell (undifferentiated) carcinoma. ${ }^{2,3}$ About $40 \%$ of NSCLC patients present with locally 
advanced noncurable (stage IIIb) or metastatic (stage IV) disease, ${ }^{4,5}$ for which the prognosis is poor. Less than $2 \%$ of patients with metastatic NSCLC are alive after 5 years., ${ }^{4,5}$

A biological and genetic variation of lung cancer is activating mutations in the tyrosine kinase domain of the epidermal growth factor receptor (EGFR). ${ }^{6}$ The prevalence of EGFR activating mutations in NSCLC varies by ethnicity, with rates of $10 \%-20 \%$ estimated in Caucasians and $30 \%-40 \%$ reported in Asian populations. ${ }^{2}$ NSCLC with no EGFR mutation is generally referred to as EGFR wildtype.

Standard first-line platinum doublet chemotherapy has shown a median overall survival of around $8-10$ months. ${ }^{7,8}$ First-line platinum doublet chemotherapy is the current standard of care for first-line treatment in patients with EGFR wild-type, but is recommended to be stopped at disease progression, or after 4-6 treatment cycles at the latest, due to cumulative toxicity and a plateau in effectiveness. ${ }^{2,3}$ Following discontinuation of chemotherapy, most patients experience disease progression within $2-3$ months, ${ }^{9,10}$ after which second-line treatment is recommended. ${ }^{2,3}$ Studies have suggested that $30 \%-50 \%$ of patients do not receive secondline treatment due to rapid disease progression, increased symptom burden, and decreasing performance status. ${ }^{10}$ Hence the use of active maintenance therapy introduced immediately after first-line platinum doublet chemotherapy in patients with complete, partial, or stable disease response to treatment has been proposed. ${ }^{2,3}$ One maintenance treatment option is erlotinib, indicated in squamous and nonsquamous cell metastatic NSCLC after 4-6 cycles of platinum-based chemotherapy. ${ }^{11,12}$ Besides erlotinib, pemetrexed is the only other switch first-line maintenance option, but is only indicated for patients with nonsquamous cell disease.

Erlotinib is an EGFR tyrosine kinase inhibitor. Its efficacy as first-line maintenance therapy has been established in the randomized, multicenter, placebo-controlled Phase III SATURN trial. ${ }^{13}$ The SATURN study compared first-line maintenance therapy with either erlotinib or best supportive care plus placebo $(\mathrm{n}=889)$ following four cycles of platinumbased chemotherapy in patients with locally advanced or metastatic NSCLC. Patients who had not experienced disease progression after initial chemotherapy were randomized 1:1 to receive erlotinib $150 \mathrm{mg} /$ day orally (plus best supportive care) or placebo (plus best supportive care) until disease progression, unacceptable toxicity, or death. The results demonstrated significant progression-free survival and overall survival benefits of erlotinib compared with placebo in the intention-to-treat population (progression-free survival hazard ratio [HR] 0.69, 95\% confidence interval [CI] $0.58-$ $0.82, P<0.0001$; overall survival HR $0.81,95 \%$ CI 0.70 $0.95, P=0.0088) .{ }^{13}$ The subpopulation of patients with stable disease following initial first-line chemotherapy appeared to benefit more from erlotinib than those with a previous complete or partial response, ${ }^{13}$ and it is for this stable disease group that erlotinib is indicated as a maintenance treatment in the European Union. ${ }^{11}$

Subgroup analyses of the EGFR wild-type population showed a significant progression-free and overall survival benefit (progression-free survival HR $0.78,95 \%$ CI $0.63-0.96, P=0.0185$; overall survival HR $0.77,95 \% \mathrm{CI}$ $0.61-0.97, P=0.0243) .{ }^{13}$

The cost-effectiveness of first-line maintenance erlotinib in patients with metastatic NSCLC and stable disease, including all patients irrespective of EGFR mutation status, has been demonstrated across three countries in the European Union in recent analyses. ${ }^{14}$ There has not yet been any assessment of whether the significant progression-free and overall survival benefit in EGFR wild-type patients observed in SATURN for first-line maintenance erlotinib corresponds to a cost-effective treatment regimen specifically in this patient group.

Thus, cost-effectiveness analyses were undertaken with the objective of determining the incremental costeffectiveness of first-line maintenance erlotinib compared with best supportive care in patients with EGFR wild-type metastatic NSCLC and stable disease following first-line therapy, in five European countries.

\section{Materials and methods Cost-effectiveness analysis}

A cost-effectiveness analysis using standard analytic decision methods was undertaken to assess the incremental cost per life-year gained from first-line maintenance erlotinib compared with best supportive care in patients with EGFR wildtype stable metastatic NSCLC. The model was programmed in Microsoft Excel 2003. The perspective of the analysis was that of national health care payers in five European countries, namely the UK, Germany, France, Spain, and Italy. For the base case analyses, costs, and health benefits were discounted at a $3.5 \%$ rate per annum.

\section{Model structure}

An area-under-the-curve (AUC) model (or partitioned survival model) was used, consisting of three health states, ie, progression-free survival, progression, and death (see Figure 1). Patients on first-line maintenance for EGFR 


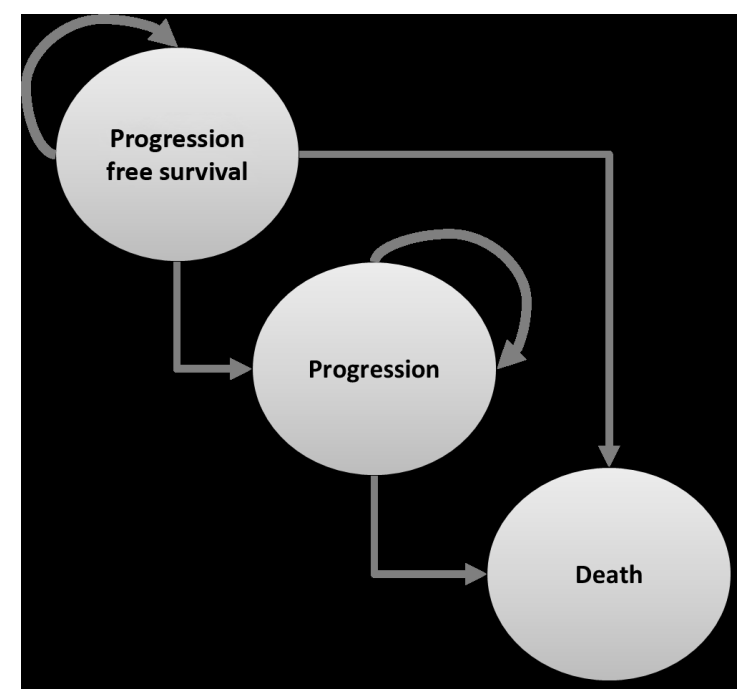

Figure I Health state transitions simulated in the cost-effectiveness model.

wild-type stable metastatic NSCLC entering the model receive either erlotinib or best supportive care and were simulated over a lifetime horizon. All patients enter the model in the "progression-free survival" health state and in each month can either progress to a "worse" health state (ie, from "progression-free survival" to "progression" or "death"; or from "progression" to "death") or remain in the same health state.

The AUC model calculates the likelihood of patients remaining in either progression-free survival or overall survival without discrete transitions using individual parametric survival curves fitted to the Kaplan-Meier curves from the SATURN study. The difference in the proportion of patients with overall and progression-free survival at any time is assumed to be the proportion of patients in the "progression" health state; death is calculated as the residual of the overall survival curve. Because an event ("progression" and "death") can occur at any time and not just at the end of each month, a half-cycle correction was applied which assumed that both costs and effects occur half way through a model cycle.

\section{Survival data}

Progression-free and overall survival data from the EGFR wild-type stable metastatic NSCLC patients in SATURN were fitted with parametric functions to extrapolate the data beyond the trial period over patients' lifetimes. Log-logistic functions $\left[1 /(1+t \times \alpha)^{\beta}\right]$ were used to simulate the timedependent $(\mathrm{t}=$ time in months $)$ probabilities of staying in the progression-free survival health state (erlotinib, $\alpha=0.097$, $\beta=2.151$; best supportive care, $\alpha=0.019, \beta=2.151$ ) and in the overall survival health state (erlotinib, $\alpha=0.009$, $\beta=1.901$; best supportive care, $\alpha=0.017, \beta=1.901$ ), respectively. These log-logistic functions provided the best fit to the data (assessed by the AUC statistic and log-likelihood ratio test) when testing various parametric functions (eg, log normal, exponential, gamma).

\section{Cost input}

For estimation of the erlotinib medication costs it was assumed that, as per the SATURN protocol, patients received a single daily dose of $150 \mathrm{mg}$ of erlotinib orally (dispensed in packs of 30 tablets). Drug costs were modeled on the basis of current ex-factory prices. As in any trial, some patients in SATURN cease treatment prior to disease progression because of treatment-related toxicities; withdrawal due to adverse events occurred in 20 patients $(5 \%)$ in the erlotinib group versus seven patients $(2 \%)$ in the best supportive care group. ${ }^{13}$ In the base case, erlotinib treatment was assumed for the time from when patients enter the model until treatment cessation, based on information from the SATURN trial. The potential costs of administering the oral drug erlotinib was considered to be small, with negligible total cost impact, and were thus not taken into account.

The costs of treating adverse events associated with erlotinib were modeled on the basis of incidence rates of adverse events $\geq$ grade 3 with a frequency $\geq 1 \%$ observed in the SATURN stable disease population prior to disease progression, ie, diarrhea (1.6\%) and rash (5.2\%). All adverse events $\geq$ grade 3 occurred in the erlotinib arm; in the placebo arm, no grade 3 or higher events were observed and thus adverse event costs were not considered for best supportive care. Incidence rates were multiplied with unit cost per adverse event episode to derive an average adverse event treatment cost, which was applied in the first model cycle (see Table 1).

For patients experiencing disease progression during the simulation, it was assumed that $73 \%$ received active second-line treatment which was the proportion seen in the SATURN trial. Active treatment with docetaxel for 90 days was assumed (reflecting the median therapy duration in major randomized controlled Phase III trials ${ }^{15-17}$ ), the costs of which were assessed on the basis of current ex-factory prices.

\section{Sensitivity analyses}

Model inputs and assumptions for the base case analysis were tested in both deterministic and probabilistic sensitivity analyses that investigated the impact of changes in key input parameters. Univariate deterministic sensitivity analyses were undertaken varying the following cost variables one 
Table I Input values used in the cost-effectiveness model analyses for the five European countries ${ }^{\mathrm{a}}$

\begin{tabular}{|c|c|c|c|c|c|}
\hline Input value & $\mathbf{U K}^{\mathrm{b}}$ & Germany & France & Spain & Italy \\
\hline Erlotinib cost $(30 \times 150 \mathrm{mg} \text { pack })^{\mathrm{c}}$ & $€ 1902$ & $€ 2309$ & $€ 2129$ & $€ 2.045$ & $€ 1963$ \\
\hline Administration erlotinib/month & $€ 0(€ 0 ; € 50)$ & $€ 0(€ 0 ; € 50)$ & $€ 0(€ 0 ; € 50)$ & $€ 0(€ 0 ; € 50)$ & $€ 0(€ 0 ; € 50)$ \\
\hline Diarrhea $^{d}$ & $€ 305$ & $€ 186$ & $€ 2632$ & $€ 264$ & $€ 394$ \\
\hline Rash $^{d}$ & $€ 150$ & $€|9|$ & $€ 2 \mid 4$ & $€ \mid 4$ & $€ 8$ \\
\hline Mean erlotinib adverse event costs ${ }^{\mathrm{e}}$ & $€ 13$ & $€ 13$ & $€ 54$ & $€ 5$ & $€ 7$ \\
\hline \multirow[t]{2}{*}{ Cost of second-line treatment ${ }^{f}$} & $€ 3803$ & $€ 5193$ & $€ 4638$ & $€ 3656$ & $€ 3431$ \\
\hline & $(€ 3043 ; € 4 \mid 84)$ & $(€ 4|54 ; € 57| 2)$ & $(€ 37|0 ; € 5| 02)$ & $(€ 2925 ; € 4022)$ & $(€ 2745 ; € 3774)$ \\
\hline Discount rate costs and effects & $3.5 \%(0 \%, 6 \%)$ & $3.5 \%(0 \%, 6 \%)$ & $3.5 \%(0 \%, 6 \%)$ & $3.5 \%(0 \%, 6 \%)$ & $3.5 \%(0 \%, 6 \%)$ \\
\hline
\end{tabular}

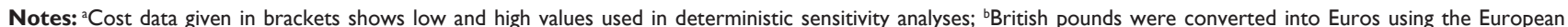
Central Bank reference exchange rate 2010 (I.1657); 'Country-specific ex-factory prices; UK, British National Formulary (BNF 58); Germany Lauertaxe May I5, 20I0; France, email communication; Spain, General Spanish Council of Pharmacists BOT database of pharmaceutical prices; Italy, L'informatore farmaceutico 20I0; 'Costs were assessed by expert interviews; ${ }^{\circ}$ Calculated on the basis of the incidence of $\geq$ grade 3 event frequencies for diarrhea and rash and the unit costs above; f 90 -day treatment with docetaxel, based on ex-factory prices December 2010 (France August 20I0), high/low values are $+10 \% /-20 \%$.

by one, ie, administration costs, second-line treatment costs, discount rates for effects, and costs (see Table 1 for parameter values tested). The base case setting on treatment duration of erlotinib (until treatment cessation) was tested by assuming treatment until disease progression (during the whole period of progression-free survival).

A probabilistic sensitivity analysis was also conducted to determine the overall influence of uncertainty within the model. Distributions around point estimates of key variables were defined that reflect parameter uncertainty. A secondorder Monte Carlo simulation with 1000 iterations was run, drawing random values simultaneously from the predefined distributions. One thousand iterations were chosen because these provide a sufficiently high number to produce stable probabilistic health and economic outcomes, comparable with results of deterministic analyses. The following types of distributions were defined: gamma distributions that account for the impossibility of negative costs and which simulate potential high cost outliers (for cost of administering erlotinib, adverse event costs, and costs of second-line treatment), beta distribution generally used to describe proportions (for the proportion of patients receiving second-line treatment), and using Cholesky decomposition of the variance-covariance matrix within log-logistic survival functions (for progressionfree survival and overall survival models).

\section{Results}

In patients with stable EGFR wild-type metastatic NSCLC, the model simulations showed that first-line maintenance erlotinib compared with best supportive care, resulted in 4.6 months of life gained (17.8 months for erlotinib versus 13.2 months for best supportive care); and 1.1 months of life without progression gained (4.3 months for erlotinib versus 3.2 months for best supportive care). The analyses showed total costs of erlotinib ranging from $€ 10,542$ (Italy) to $€ 13,203$ (Germany) and total costs of best supportive care from $€ 2393$ (Italy) to $€ 3623$ (Germany), resulting in total incremental costs ranging from $€ 7897$ (the UK) to $€ 9580$ (Germany). Mean incremental costs per life-year gained with first-line maintenance erlotinib compared with best supportive care in stable EGFR wild-type metastatic NSCLC ranged between $€ 20,711$ (the UK) and $€ 25,124$ (Germany); all well within ranges typically considered as cost-effective (Table 2).

Sensitivity analyses results did not vary much because of changes in input values. The most sensitive variables were the discount rate for effects and duration of treatment. Lowest incremental cost-effectiveness ratios were found when lowering the discount rate for the treatment effect to $0 \%$, highest values when assuming erlotinib treatment until progression and not until treatment cessation (Table 2).

The cost-effectiveness acceptability curves resulting from probabilistic sensitivity analyses show that erlotinib would be considered cost-effective across the range of possible willingness-to-pay thresholds (see Figure 2). Looking exemplarily at a cost per life-year gained threshold of $€ 50,000$, the probability that erlotinib is cost-effective is $93.6 \%$ in the UK, $91.0 \%$ in Germany, $91.7 \%$ in France, $92.1 \%$ in Spain, and $92.9 \%$ in Italy.

\section{Discussion}

This analysis is the first to assess the cost-effectiveness of erlotinib as first-line maintenance treatment in patients with stable EGFR wild-type metastatic NSCLC. The results of the modeled analyses demonstrate an incremental costs per life year gained ranging between $€ 20,711$ and $€ 25,124$ comparing first-line maintenance erlotinib with best supportive care in five European countries (UK, Germany, France, Spain and Italy), and thus well within a range generally considered as cost-effective. Deterministic and probabilistic sensitivity 
Table 2 Results of cost-effectiveness analyses: base case and sensitivity analyses for five European countries

\begin{tabular}{|c|c|c|c|c|c|c|c|c|c|c|}
\hline \multirow{2}{*}{$\begin{array}{l}\text { Country } \\
\text { Comparators }\end{array}$} & \multicolumn{2}{|l|}{ UK } & \multicolumn{2}{|c|}{ Germany } & \multicolumn{2}{|l|}{ France } & \multicolumn{2}{|l|}{ Spain } & \multicolumn{2}{|l|}{ Italy } \\
\hline & ERL & BSC & ERL & BSC & ERL & BSC & ERL & BSC & ERL & BSC \\
\hline \multicolumn{11}{|c|}{ Base case: mean overall survival and costs } \\
\hline Mean years without & 0.36 & 0.26 & 0.36 & 0.26 & 0.36 & 0.26 & 0.36 & 0.26 & 0.36 & 0.26 \\
\hline progression (months) & $(4.29)$ & $(3.15)$ & $(4.29)$ & $(3.15)$ & $(4.29)$ & $(3.15)$ & $(4.29)$ & $(3.15)$ & $(4.29)$ & $(3.15)$ \\
\hline \multirow[t]{2}{*}{ Mean life-years (months) } & 1.49 & 1.10 & 1.49 & 1.10 & 1.49 & 1.10 & 1.49 & 1.10 & 1.49 & 1.10 \\
\hline & $(17.82)$ & $(13.24)$ & $(17.82)$ & $(13.24)$ & $(I 7.82)$ & (I3.24) & $(\mid 7.82)$ & $(13.24)$ & $(17.82)$ & $(13.24)$ \\
\hline Mean total cost, $€$ & $|0,55|$ & 2653 & 13,203 & 3623 & 12,109 & 3236 & 11,039 & 2551 & 10,542 & 2393 \\
\hline \multicolumn{11}{|c|}{ ICER - cost per life year gained of erlotinib versus BSC, $€$} \\
\hline Base case & $20,7 \mid I$ & & 25,124 & & 23,271 & & 22,261 & & 21,368 & \\
\hline \multicolumn{11}{|c|}{ Deterministic SA: ICER - cost per life year gained of erlotinib versus BSC, $€$} \\
\hline Discount rate effect $0 \%$ & 19,111 & & 23,184 & & 21,474 & & 20,542 & & 19,718 & \\
\hline Treatment duration: PFS & 23,132 & & 28,064 & & 25,981 & & 24,865 & & 23,867 & \\
\hline
\end{tabular}

Abbreviations: ERL, erlotinib; BSC, best supportive care; ICER, incremental cost-effectiveness ratio; SA, sensitivity analyses; PFS, progression-free survival.

analyses confirmed these findings, indicating that erlotinib first-line maintenance in stable EGFR wild-type metastatic NSCLC translates into improved overall survival and costeffectiveness across jurisdictions in Europe. These analyses are based on empiric progression-free and overall survival data from the SATURN trial, and several parametric survival models were tested to identify the model with the best fit to the data.

In the SATURN trial, biomarker analyses for EGFR status showed that first-line maintenance erlotinib was efficacious irrespective of the EGFR status. ${ }^{13}$ However, for the EGFR activating mutation-positive stable metastatic NSCLC population, patient numbers in SATURN were too small $(\mathrm{n}=20)$ to fit survival models around the data reliably, and hence model the cost-effectiveness.

The cost-effectiveness of first-line maintenance erlotinib versus best supportive care stable metastatic NSCLC population, including all patients irrespective of EGFR mutation status, throughout European countries has recently been demonstrated in modeled analyses by Vergnenegre et al. ${ }^{14}$ This analysis found that first-line maintenance erlotinib resulted in incremental costs per life year gained of between $€ 27,885$

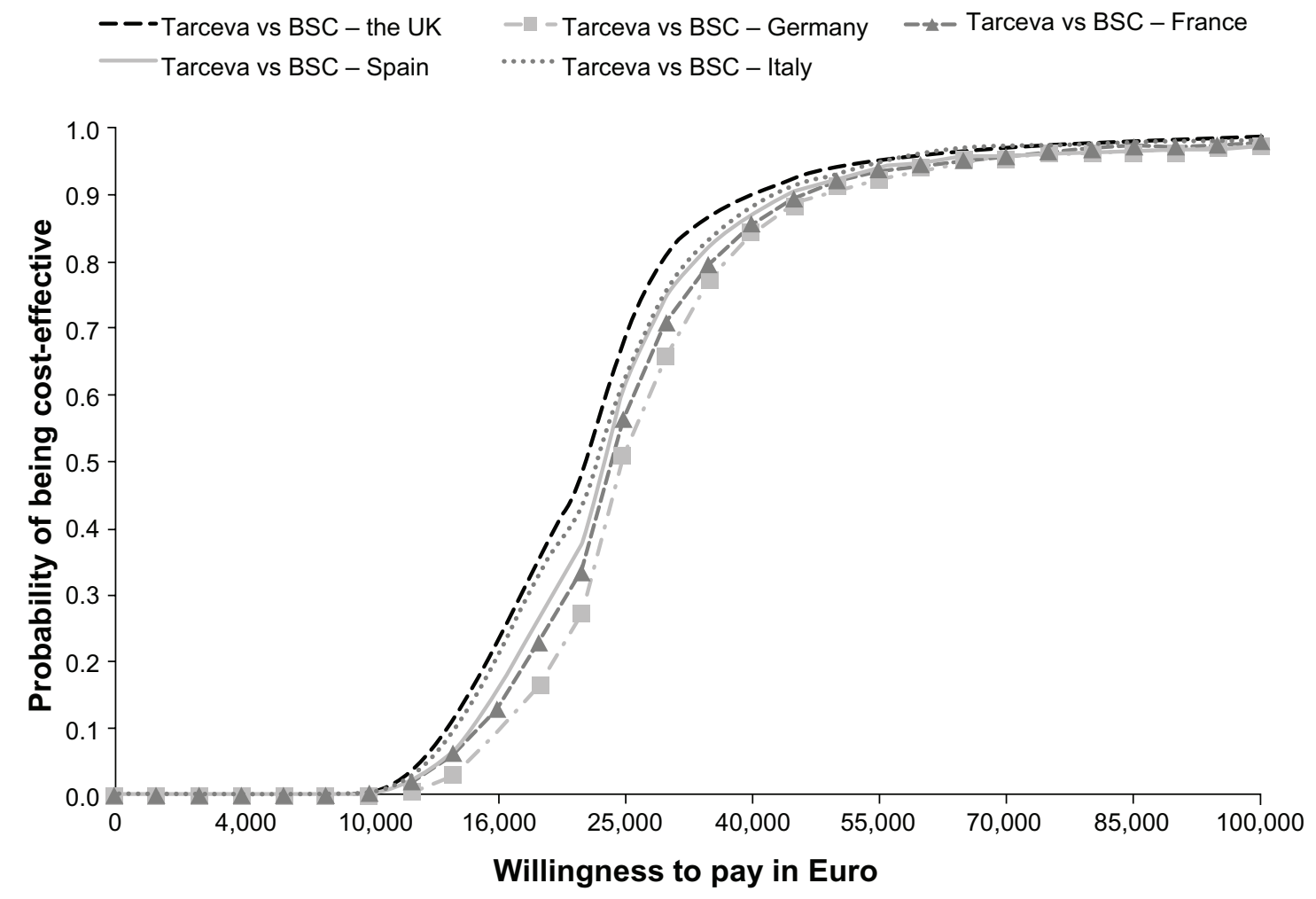

Figure 2 Results of probabilistic sensitivity analyses: cost-effectiveness acceptability curves for all five countries. 
and $€ 39,783$. Survival in this analysis was also modeled on the basis of the SATURN data. However, as the overall SATURN stable disease population showed a lower overall survival treatment effect than the stable EGFR wild-type subpopulation, the incremental cost-effectiveness ratios reported by Vergnenegre et al were higher than in our analyses. The lower survival in the overall stable disease population could potentially be explained by the fact that $50 \%$ of patients in SATURN were of either "indeterminate" or "missing" EGFR mutation status, with $44 \%$ being wild-type and only $6 \%$ having EGFR activating mutations. This low proportion of patients tested for EGFR status may explain the observed variations in results.

The analyses presented are based on robust and transparent modeling and costing methods. Ex-factory drug prices were assumed for both erlotinib and secondline treatment (docetaxel), without considering potential pricing schemes available in the countries included. Administration costs were not considered for the base case analysis because they were regarded as negligible, given that erlotinib is administered orally. Sensitivity analyses varying administration costs have confirmed this assumption, because this parameter was not found to influence the results significantly. Costs of best supportive care were not considered in either the erlotinib or the best supportive care comparison group, because these costs are likely to be similar in both and thus their exclusion should not greatly affect the differences in costs and in the incremental cost-effectiveness ratios observed.

Assumptions on second-line treatment had to be made (for patients experiencing disease progression in the course of the simulation), whereby 90 days of docetaxel treatment was assumed for a proportion of patients. Other more expensive second-line agents such as pemetrexed are increasingly being utilized in first-line treatment, which was the reason for using docetaxel to estimate second-line treatment costs. Even higher second-line treatment costs would probably not affect incremental cost-effectiveness ratios, because these costs are likely to be comparable between the erlotinib and the best supportive care groups.

In addition, given that patients receiving erlotinib might progress later than patients in best supportive care, discounting costs might actually lead to higher overall second-line costs accrued in the best supportive care arm than in the erlotinib arm. This effect can be observed in the model, where second-line costs are spread pro rata across the progressive disease period. In order to test the impact on results of uncertain variables, all uncertain input parameters were tested in deterministic and probabilistic sensitivity analyses that demonstrated the robustness of base case results.

Besides erlotinib, other first-line maintenance options include targeted agents, such as bevacizumab, added to first-line platinum doublet chemotherapy and then continued until disease progression (continuous maintenance therapy). ${ }^{2,3}$ Besides erlotinib, pemetrexed is the only other switch first-line maintenance option, but it is only indicated for nonsquamous cell patients, ${ }^{18}$ whereas erlotinib is indicated in both nonsquamous and squamous cell patients. ${ }^{11}$

A recent population-matched indirect comparison of erlotinib and pemetrexed considering the intention-to-treat population of the SATURN and JMEN trials has found both treatments to be similarly efficacious. ${ }^{19}$ In addition, Nuijten et al have demonstrated that erlotinib is less costly than pemetrexed. ${ }^{20}$ Further considering other demonstrated advantages of erlotinib in terms of tolerability, administration, and patient convenience, ${ }^{19}$ erlotinib appears to be the preferred treatment option over pemetrexed. Considering this, and taking into account that pemetrexed is only indicated in nonsquamous patients, whereas most EGFR wild-type patients are squamous, we did not regard pemetrexed as an appropriate comparator and therefore focused our analysis on comparing erlotinib with best supportive care.

In first-line NSCLC therapy, the choice of therapy according to EGFR mutation status has been proposed as a way to target treatment to the patient group that is most likely to benefit from erlotinib therapy. For example, somatic mutations in the EGFR gene have been proposed as the most robust biomarkers for EGFR-targeted choice of therapy in first-line NSCLC therapy. ${ }^{21}$ However, for first-line maintenance, second-line and third-line therapy, there is no evidence supporting such an EGFR biomarker-based patient selection scheme for erlotinib. ${ }^{21,22}$ According to the clinical perspective shown above, our health economic analyses do not provide any indication for using EGFR activating mutation status as a predictor of more or less cost-effective subgroups for treatment with erlotinib, because patients with wild-type EGFR appear to benefit from first-line maintenance erlotinib at reasonable cost.

\section{Conclusion}

First-line erlotinib maintenance treatment is efficacious and represents good value for money compared with best supportive care, regardless of country setting, in patients with metastatic NSCLC and stable wild-type EGFR according to a cost-effectiveness model based on the Phase III SATURN trial data. 


\section{Disclosure}

The authors report no conflicts of interest in this work.

\section{References}

1. Ferlay J, Shin HR, Bray F, Forman D, Mathers C, Parkin DM. Estimates of worldwide burden of cancer in 2008: GLOBOCAN 2008. Int J Cancer. 2010;127(12):2893-2917.

2. NCCN Guidelines - Clinical Practice Guidelines in Oncology. Non-small cell lung cancer. Available at: http://www.nccn.org/ professionals/physician_gls/pdf/nscl.pdf. Accessed May 16, 2012.

3. D'Addario G, Fruh M, Reck M, Baumann P, Klepetko W, Felip E. Metastatic non-small-cell lung cancer: ESMO clinical practice guidelines for diagnosis, treatment and follow-up. Ann Oncol. 2010;21(5): 116-119.

4. Mountain CF. The international system for staging lung cancer. Semin Surg Oncol. 2000;18(2):106-115.

5. Goldstraw P, Crowley J, Chansky K, et al. The IASLC Lung Cancer Staging Project: proposals for the revision of the TNM stage groupings in the forthcoming (seventh) edition of the TNM classification of malignant tumours. J Thorac Oncol. 2007;2(8):706-714.

6. Gazdar AF. Activating and resistance mutations of EGFR in non-smallcell lung cancer: role in clinical response to EGFR tyrosine kinase inhibitors. Oncogene. 2009;28(1):24-31.

7. Ramalingam S, Belani C. Systemic chemotherapy for advanced non-small cell lung cancer: recent advances and future directions. Oncologist. 2008;13(1):5-13.

8. Sandler A, Gray R, Perry MC, et al. Paclitaxel-carboplatin alone or with bevacizumab for non-small-cell lung cancer. $N$ Engl J Med. 2006;355(24):2542-2550.

9. Brodowicz T, Krzakowski M, Zwitter M, et al. Cisplatin and gemcitabine first-line chemotherapy followed by maintenance gemcitabine or best supportive care in advanced non-small cell lung cancer: a Phase III trial. Lung Cancer. 2006;52(2):155-163.

10. Fidias PM, Dakhil SR, Lyss AP, et al. Phase III study of immediate compared with delayed docetaxel after front-line therapy with gemcitabine plus carboplatin in advanced non-small-cell lung cancer. J Clin Oncol. 2009;27(4):591-598.

11. European Medicines Agency. Tarceva (erlotinib) - EU label and approval. Available at: http://www.ema.europa.eu/docs/en_GB/ document_library/EPAR_-_Product_Information/human/000618/ WC500033994.pdf. Accessed May 16, 2012.

12. US Food and Drug Administration. Tarceva (erlotinib) - FDA label and approval. Available at: http://www.accessdata.fda.gov/drugsatfda_docs/ label/2010/021743s14s16lbl.pdf. Accessed May 16, 2012.
13. Cappuzzo F, Ciuleanu T, Stelmakh L, et al. Erlotinib as maintenance treatment in advanced non-small-cell lung cancer: a multicentre, randomised, placebo-controlled Phase 3 study. Lancet Oncol. 2010;11(6):521-529.

14. Vergnenègre A, Ray J, Chouaid C, et al. Cross-market cost-effectiveness analysis of erlotinib as first-line maintenance treatment for patients with stable non-small cell lung cancer. Clinicoecon Outcomes Res. 2012;4(1):31-37.

15. Shepherd FA, Dancey J, Ramlau R, et al. Prospective randomized trial of docetaxel versus best supportive care in patients with non-small-cell lung cancer previously treated with platinum-based chemotherapy. J Clin Oncol. 2000;18(10):2095-2103.

16. Shepherd FA, Rodrigues PJ, Ciuleanu T, et al. Erlotinib in previously treated non-small-cell lung cancer. N Engl J Med. 2005;353(2): 123-132.

17. Hanna N, Shepherd FA, Fossella FV, et al. Randomized Phase III trial of pemetrexed versus docetaxel in patients with non-small-cell lung cancer previously treated with chemotherapy. J Clin Oncol. 2004;22(9):1589-1597.

18. European Medicines Agency. Alimta (pemetrexed) - EU label and approval. Available at: http://www.ema.europa.eu/docs/en_GB/ document_library/EPAR_-_Product_Information/human/000564/ WC500025611.pdf. Accessed May 16, 2012.

19. Casciano R, Bischoff H, Nuijten M, Malangone E, Ray J. Maintenance erlotinib versus pemetrexed for the treatment of non-small cell lung cancer: indirect comparison applying real-life outcomes. Proceedings of the International Society for Pharmacoeconomics and Outcomes Research 13th Annual European Congress, November 6-9, 2010; Prague, Czech Republic.

20. Nuijten MJ, de Castro CJ, Chouaid C, et al. A cross-market cost comparison of erlotinib versus pemetrexed for first-line maintenance treatment of patients with locally advanced or metastatic non-small-cell lung cancer. Lung Cancer. 2012;76(3):465-471.

21. Ontario Health Technology Advisory Committee. Epidermal growth factor receptor (EGFR) mutation testing for prediction of response to EGFR-targeting tyrosine kinase inhibitor (TKI) drugs in patients with advanced non-small-cell lung cancer. Presentedat the Ontario Health Technology Advisory committee in August, 2010. Available at: http:/www.health.gov.on.ca/english/providers/program/ohtac/tech/ recommend/rec_EGFR_20101209.pdf. Accessed May 16, 2012.

22. Bradbury PA, Tu D, Seymour L, et al. Economic analysis: randomized placebo-controlled clinical trial of erlotinib in advanced non-small cell lung cancer. J Natl Cancer Inst. 2010;102(5):298-306.
ClinicoEconomics and Outcomes Research

\section{Publish your work in this journal}

ClinicoEconomics \& Outcomes Research is an international, peerreviewed open-access journal focusing on Health Technology Assessment, Pharmacoeconomics and Outcomes Research in the areas of diagnosis, medical devices, and clinical, surgical and pharmacological intervention. The economic impact of health policy and health systems

\section{Dovepress}

organization also constitute important areas of coverage. The manuscript management system is completely online and includes a very quick and fair peer-review system, which is all easy to use. Visit http://www.dovepress.com/testimonials.php to read real quotes from published authors. 